ISSN 0103-7013

Psicol. Argum., Curitiba, v. 29, n. 66, p. 361-371, jul./set. 2011

Licenciado sob uma Licença Creative Commons

\title{
Julgamento de adolescentes sobre pessoas desconhecidas fumantes e não fumantes ${ }^{1}$
}

\author{
Teenagers' judgment of unknown smokers and nonsmokers
}

\author{
Paula Vanessa Pestana Simão ${ }^{[a]}$, Sandro Caramaschi ${ }^{[\mathrm{b}]}$
}

[a] Mestre em Psicologia pela Universidade Estadual de São Paulo (UNESP), Departamento de Psicologia, Faculdade de Ciências, Universidade Estadual de São Paulo (UNESP), Bauru, SP - Brasil.

[b] Doutor pelo Instituto de Psicologia, Universidade de São Paulo (USP), Departamento de Psicologia, Faculdade de Ciências, Universidade Estadual de São Paulo (UNESP), Bauru, SP - Brasil, e-mail: caramas@fc.unesp.br

\section{Resumo}

A adesão ao hábito de fumar vem preocupando especialistas em saúde e educadores, principalmente no que diz respeito a adolescentes. Entretanto, poucos estudos se dedicam a investigar a perspectiva dos próprios jovens acerca das pessoas que fumam. Neste estudo, a percepção de adolescentes alunos do ensino médio sobre pessoas desconhecidas fumantes e não fumantes foi investigada. A amostra foi constituída de 100 alunos (50 do sexo masculino e 50 do feminino) com idades entre 16 e 21 anos. A coleta de dados foi feita mediante apresentação de fotos de modelos (um homem e uma mulher) em duas versões: com cigarro e sem cigarro na mão. A atribuição de características foi feita por meio de um instrumento especificamente construído para esta pesquisa, a partir da pontuação em escalas bipolares de 15 características. A análise dos dados nos permitiu constatar que, de uma forma geral, as pessoas sem cigarro são julgadas de maneira mais favorável em $86 \%$ das características investigadas. Foram verificadas algumas diferenças na forma como homens e mulheres atribuem valores aos modelos com e sem cigarro nas características construtividade, saúde, "legal", lealdade, calma, otimismo, discrição, confiabilidade, bumildade e sabedoria. Os dados permitem verificar a opinião de jovens a respeito da aparência de pessoas fumantes, podendo-se dessa forma direcionar esforços mais eficientes ao controle do tabagismo.

Palavras-chave: Adolescente. Tabagismo. Comportamento. Percepção.

1 Apoio financeiro: Bolsa Fundação de Amparo à Pesquisa do Estado de São Paulo. 


\begin{abstract}
The addiction to the habit of smoking is a main worry to health experts and educators, mainly with respect to adolescents. However, few studies are conducted with the purpose of investigating how the teenagers themselves see and judge people who smoke. In this work, the perception of adolescent high school students of unknown smokers and nonsmokers was investigated. The sample constituted of 100 students (50 male and 50 female) aged from 16 to 21 years old. The data were collected by presenting photographs of models (a man and a woman) in two versions: holding a cigarette or not. The attribution of characteristics was accomplished through an instrument specifically elaborated for this research, through scores on bipolar scales of 15 characteristics. An analysis of data allowed us to verify that, in general, people without a cigarette were more favorably judged in $86 \%$ of the characteristics investigated. Data also showed some differences between male and female judgments of models with or without a cigarette concerning the following characteristics: constructiveness, health, niceness, loyalty, calmness, optimism, discretion, reliability, humility and wisdom. This research allowed us to verify the opinion of teenagers about the appearance of smokers, which could contribute to directing the efforts towards smoking control more efficiently.
\end{abstract}

Keywords: Adolescents. Smoking. Behavior. Perception.

\section{Introdução}

Estatísticas da Organização Mundial da Saúde (OMS) indicam que nos primeiros 25 anos do século XXI o vício do cigarro matará mais de 100 milhões de pessoas no mundo. A dependência física (vício) causada pelo fumo é maior do que a gerada pelo uso de drogas como cocaína e heroína, o que pode ser explicado em função da alta frequência com que o cigarro é consumido, e também pelo fácil acesso de qualquer pessoa em qualquer idade (Dedobbeleer, Beland, Contadriopoulos \& Adrian, 2004).

O constante aumento do consumo de cigarro permite concluir que os preceitos científicos sobre as doenças causadas pelo tabagismo não têm sido uma contrapropaganda eficiente. As propagandas sempre contribuíram para tornar o cigarro símbolo de posição social, sedução, beleza, liberdade, entre outros. Para tal, utiliza-se a associação de elementos estritamente positivos para a maioria das pessoas (como belas paisagens, esportes radicais, pessoas bonitas) aos produtos que se pretende vender. Um estudo realizado com adolescentes brasileiros demonstrou que a manipulação realizada pela propaganda não é percebida pelo seu principal público-alvo (Machado, Gomide \& Caldeira, 2004).

A indústria da propaganda continua contribuindo fortemente para a propagação do cigarro, ludibriando seu público-alvo com divulgações enganosas de tamanha eficiência que, além de propiciar o aumento das vendas do produto, neutralizam ou invalidam as campanhas informativas existentes a respeito dos prejuízos causados pelo tabagismo (Machado et al., 2004).

Isso pode estar relacionado ao fato de as campanhas informativas trabalharem com o saber, atuando numa dimensão eminentemente cognitiva e racional, enquanto as campanhas publicitárias o fazem com o sentir, que age na vertente sensorial e emocional. Ou seja, as mensagens contra o tabagismo normalmente têm como enfoque tão somente a informação, que, em razão da maneira concreta como é fornecida, não instiga atitudes de mudança de conduta nas pessoas. Portanto, essas informações até transmitem o conhecimento, porém não são suficientes para mudar comportamentos em relação ao tabagismo. Já as propagandas utilizam vários recursos, como a criação de imagens e situações que seduzem as pessoas, propiciando sensações de bem-estar que poderão ser associadas automaticamente ao uso do cigarro (Rosendahl, Galanti, Gilljam \& Ahlbom, 2005).

As campanhas publicitárias atingem principalmente os jovens, e o mais preocupante é que as estatísticas mostram que a procura e iniciação ao tabagismo têm se dado cada vez mais precocemente, o que leva à necessidade de uma reflexão acerca dos fatores responsáveis por isso, tais como a autoimagem do jovem (Falomir-Pichastor, Mugny, Invernizzi, Di-Palma \& Estrada, 2007). Esses fatores variam de um indivíduo para outro, ou seja, são 
biopsicossociais (Silva, Silva \& Botelho, 2008), e englobam desde apreciação das alterações fisiológicas causadas pelo fumo (Meliska \& Gilbert, 1991), aspectos familiares (Fraga, Ramos \& Barros, 2008), alívio ou fuga de conflitos psicológicos (Rodriguez \& Audrain-McGovern, 2005), até maior aceitação social (Forster, Chen, Blaine, Perry \& Toomey, 2003). Essas relações interpessoais gerarão crenças e expectativas nesses indivíduos diretamente ligadas ao conceito que eles irão desenvolver a respeito do hábito de fumar, bem como das pessoas que fumam (Ogden \& Nicolli, 1997).

Nesse sentido, o indivíduo na adolescência é um potencial consumidor do cigarro, o que pode ser justificado em virtude das várias modificações presentes nessa fase da vida. Sabe-se que a adolescência é caracterizada como um período presente no desenvolvimento humano desde a puberdade até a fase de adulto jovem, podendo sofrer variações de um indivíduo para o outro, bem como de acordo com sua cultura e posição socioeconômica (Gomes \& Caramaschi, 2007). Esse período é marcado por intensas transformações fisiológicas caracterizadas por alterações hormonais e modificações corporais (Brasil, Tavano, Caramaschi \& Rodrigues, 2007), além de alterações psicológicas, evidenciadas pela presença de conflitos sociais e determinadas pelo aumento de responsabilidades demandadas dos novos papéis sociais (White, Hill \& Hopper, 1996; Gilpin, Lee \& Pierce, 2004).

Nessa fase do desenvolvimento humano há uma procura mais intensa por grupos aos quais se possa e queira pertencer ou seguir (Paek \& Gunter, 2007). Surge a tendência de questionar e romper com as regras familiares, aumentando consideravelmente o desejo de adotar um estilo original (Hoffman, Sussman, Unger \& Valente, 2006). Assim, o risco se concretiza, já que o adolescente pauta suas ações no pensamento mágico (presente na infância e mantido na puberdade) caracterizado por uma sensação de onipotência-fazendo o que quiser e experimentando tudo. É nesse momento que há um alto número de adolescentes que entram em contato com o cigarro, entre outras novidades (Soldz, Huyser \& Dorsey, 2003).

Os fumantes frequentemente se engajam em vários comportamentos ou discursos que visam a justificar ou reduzir sua dissonância cognitiva (Festinger, 1962/1975), que está presente nos casos de tabagismo porque é de conhecimento público que o cigarro causa males à saúde, bem como incomoda a maior parte dos não fumantes. Por estar em desacordo com essa realidade, a opção do indivíduo por fumar produz a dissonância. Ou seja, há uma contradição entre o que ele faz (fumar) e as informações que possui sobre o assunto (fumar não é bom). Nessa situação, duas alternativas são possíveis: o indivíduo pode parar de fumar ou desenvolver argumentos e percepções que justifiquem seu hábito (Tomar \& Hatsukami, 2007; Gibson, 1997).

$\mathrm{O}$ adolescente geralmente nega o vício, alegando que pode controlar o uso do cigarro e parar quando assim o desejar (Halpern-Felsher, Biehl, Kropp \& Rubinstein, 2004). Desse modo, ele acredita que os males causados pelo fumo não o atingirão, pois deixará de fumar assim que perceber que isso lhe está fazendo mal. Com isso, poderá tornar-se cada vez mais dependente da nicotina até sentir-se dominado pelo hábito.

Percebe-se, portanto, que o tabagismo é um problema de saúde pública, e os trabalhos que poderão surtir os maiores efeitos são os de caráter preventivo. Diante disso, o Ministério da Saúde, aliado ao Ministério da Educação, vem atuando para que se realize um trabalho educativo, capaz de alertar a população no que diz respeito aos males causados pelo tabagismo. As escolas devem abordar temas relacionados à saúde nas diversas disciplinas e entre eles deve-se incluir o uso de cigarro, álcool, cocaína e outras drogas em geral. São os chamados "temas transversais" nos Parâmetros Curriculares Nacionais.

As pesquisas relacionadas ao tabagismo entre adolescentes, em sua maioria, visam a quantificar o número de jovens fumantes, a idade em que começaram a prática ou os fatores que levaram esses jovens a iniciá-la (Simões, 1990; Silva, Silva \& Botelho, 2008). Esses estudos têm fornecido dados importantes para investigações sobre o hábito de fumar - no entanto, são poucos os trabalhos que avaliam o julgamento que os jovens fazem sobre os fumantes, adentrando sua dimensão emocional (Gibson, 1997; Piko, Bak \& Gibbons, 2007).

Ao saber quais impressões (positivas ou negativas) os jovens possuem sobre os fumantes, pode-se preparar campanhas de conscientização mais eficazes. Deve-se investigar quais são as expectativas e julgamentos que os jovens apresentam em relação ao hábito de fumar, pois se não houver uma modificação desses elementos, quaisquer trabalhos 
educativos em relação ao tabagismo correm o risco de serem ineficazes (Machado et al., 2004) ou de criarem estratégias de resistência (Spink, 2009).

$\mathrm{Na}$ nossa opinião, qualquer tipo de abordagem direta em relação aos adolescentes, perguntando-lhes suas opiniões sobre o tabagismo, produziria uma série de respostas estereotipadas do discurso oficial. Nossa prerrogativa, neste trabalho, foi abordar a perspectiva de jovens estudantes sobre o hábito de fumar, sem, no entanto, referirmo-nos diretamente ao assunto.

Dessa forma, propusemo-nos a investigar a percepção dos jovens acerca de pessoas desconhecidas no intuito de verificar quais são as características atribuídas predominantemente a indivíduos que fumam.

Concomitantemente, procuramos verificar a influência do sexo do modelo no julgamento social do hábito de fumar, verificando possíveis diferenças na maneira como o modelo é julgado de acordo com o gênero.

Outra proposta foi verificar a importância do sexo do jovem que realiza a avaliação, investigando se esse fator estabelece alguma relação com a maneira como ele julga os modelos masculinos e femininos.

Finalmente, propusemo-nos a investigar a interferência do fato de os juizes serem fumantes ou não na atribuição de características aos modelos apresentados.

\section{Método}

\section{Material visual}

Foram produzidas imagens por meio de fotografias em diapositivos de alunos universitários, sendo um indivíduo do sexo masculino e um do sexo feminino, com idades aproximadas de 20 anos. Os modelos foram fotografados sentados, em posição relaxada e com expressão facial neutra. Duas fotos de cada indivíduo foram produzidas: em uma delas o modelo era apresentado com um cigarro entre os dedos e na outra sem o cigarro, sendo mantidas todas as outras características como local, iluminação, roupas, postura corporal e expressão facial. Dessa forma, podemos inferir que qualquer diferença verificada no julgamento dos modelos por grupos independentes deve ser decorrente da presença do cigarro.

\section{Pré-teste}

Primeiramente foi elaborado um instrumento constituído por um questionário de diferencial semântico, com 30 características bipolares de 10 pontos, que foi utilizado para o pré-teste. Tal procedimento consistiu em apresentar os dispositivos com as fotos dos modelos individualmente a duas turmas de 30 alunos universitários, e aplicar o instrumento com 30 características bipolares para cada modelo apresentado.Os participantes assinaram termo de consentimento livre e esclarecido, sendo então instruídos para que atribuíssem valores próximos à extremidade das características que julgassem mais relacionadas com o modelo. Cada grupo de sujeitos observou e avaliou um indivíduo de cada sexo, mas nunca em condições diferentes (com cigarro e sem cigarro). Os dados do pré-teste foram tabulados e, a partir disso, foram calculadas as médias para cada característica de cada modelo.

Foram então comparadas as médias das características atribuídas ao modelo com cigarro e do mesmo modelo sem cigarro. As que apresentaram menor variação (diferença inferior a 0,5 ) entre os modelos com e sem cigarro no julgamento dos participantes foram excluídas e, consequentemente, as 15 características que apresentaram maior variação foram selecionadas para o instrumento definitivo de pesquisa.

No instrumento definitivo utilizado, também havia questões referentes a dados pessoais dos sujeitos relativos a idade e sexo. Além desse material, foram elaboradas questões de múltipla escolha relacionadas ao consumo de cigarros pelos participantes, categorizando o uso como não usuário, esporádico ou frequente, bem como as situações em que o consumo era realizado.

\section{Participantes}

Após a definição da quantidade e especificidade dos sujeitos que seriam abordados, estabeleceu-se contato com uma escola estadual de ensino médio de Bauru, para que pudéssemos utilizar alguns de seus alunos como participantes da pesquisa. A diretora da escola autorizou a aplicação do instrumento sem restrições.

Participaram da pesquisa 100 alunos originários de quatro turmas de segundo ano do período 
noturno, sendo 50 do sexo masculino e 50 do feminino, com idades entre 16 e 21 anos.

\section{Procedimento}

Os pesquisadores abordaram as turmas em suas salas de aula. O objetivo da pesquisa não foi totalmente explicitado, dizendo-se simplesmente que se tratava de um estudo sobre aparência.

Os participantes foram informados, mediante um termo de consentimento esclarecido, acerca do caráter voluntário da participação na pesquisa, bem como da possibilidade de deixar de participar em qualquer momento da coleta de dados - no final, todos os alunos abordados decidiram participar. Os participantes menores de idade levaram os termos de consentimento para serem assinados por pais ou responsáveis, enquanto os maiores assinaram o termo de consentimento, os quais foram recolhidos no dia seguinte, antes da aplicação da pesquisa.

Durante a coleta de dados as turmas permaneceram nas salas de aula. A cada grupo foram apresentadas duas fotos (masculina e feminina) em uma das condições experimentais (com cigarro ou sem cigarro). Dessa forma, cada participante respondeu a dois questionários, um para cada modelo. Os participantes que viram um dado modelo, com ou sem cigarro, não o viram em outra condição. Cada aplicação durou em torno de 20 minutos, com tempo livre para o preenchimento do instrumento - a imagem permaneceu visível durante todo o período da atividade. Ao término da apresentação das duas fotos e preenchimento das escalas de diferencial semântico, os participantes responderam o questionário sobre seus hábitos de consumo de cigarros.

Ao final da tarefa, foram apresentados aos participantes os reais objetivos da pesquisa, com os devidos agradecimentos pela colaboração.

\section{Tabulação e análise dos dados}

A partir da classificação dos dados, foi feita, para cada uma das quinze características, uma tabela contendo os quatro principais grupos (modelos masculino e feminino com e sem cigarro), de acordo com o gênero de cada grupo de juízes (femininos e masculinos). Dessa forma, foram tabuladas as respostas de cada sujeito diante das duas condições experimentais para cada característica. Então, foram calculadas as médias, as quais são apresentadas na Tabela 1.

A partir dessa classificação dos dados, foi possível investigar as três primeiras hipóteses. Para a investigação da influência do hábito de fumar dos sujeitos no julgamento, foram selecionados, a partir do questionário complementar, quais dos participantes eram ou não fumantes. Foram organizadas três categorias de respostas constituídas por pessoas que nunca fumaram (52\%); não fumam, mas já experimentaram $(28 \%)$ e que fumam frequentemente ou esporadicamente $(20 \%)$. Para fins estatísticos, foram selecionados aleatoriamente 20 indivíduos fumantes (10 de cada sexo) e um grupo de 20 participantes que nunca fumaram.

Para investigar o nível de significância das diferenças entre as médias comparadas, foram utilizados os testes não paramétricos de Wilcoxon, para dados pareados, e de Mann-Whitney, para grupos independentes.

\section{Resultados}

A pesquisa sobre atribuição de características a pessoas fumantes e não fumantes por alunos do ensino médio teve como foco principal de investigação a diferença entre os julgamentos realizados, pelos jovens, de quinze características para modelos com e sem cigarro. Estabeleceu-se por hipótese que os modelos sem cigarro seriam julgados mais positivamente do que os modelos com cigarro, os quais teriam uma avaliação mais negativa.

Tal hipótese foi lançada com base na perspectiva de que, em virtude da grande quantidade de informações sobre os males causados pelo tabagismo, as pessoas de uma maneira geral tenderiam a atribuir características mais negativas aos modelos que apresentassem o cigarro, por associá-los de uma maneira subliminar aos malefícios causados.

Outros aspectos da investigação foram formados a partir desse foco principal. Procurou-se verificar se juízes do sexo masculino julgavam mais positivamente modelos masculinos, e se juízes femininos julgavam mais positivamente modelos femininos e vice-versa.

O fato de o juiz ser fumante ou não fumante foi investigado no sentido de se verificar se tal 
variável interfere no julgamento dos modelos. Essa hipótese foi lançada para se verificar se a dissonância cognitiva (Festinger, 1962/1975) está presente no julgamento, ou seja, imagina-se que os indivíduos fumantes tenderiam a julgar mais positivamente modelos com características similares às suas para diminuir a dissonância cognitiva.

Os resultados obtidos foram dispostos em tabelas, que permitem uma melhor observação e análise. Os resultados serão apresentados focando inicialmente as diferenças sexuais dos sujeitos na atribuição de características de homens e mulheres posteriormente apresentaremos os dados relativos ao hábito de fumar dos participantes.

\section{Participantes masculinos}

A Tabela 1 demonstra que, nos julgamentos realizados por indivíduos do sexo masculino em relação ao modelo feminino, quase todas as características (87\%) tiveram valores, de acordo com as médias, maiores para o modelo sem cigarro. Os resultados tomados em conjunto por meio da prova não-paramétrica de Wilcoxon demonstraram que as atribuições ao modelo sem cigarro são significativamente maiores do que os com cigarro $(\mathrm{Z}=-3,2374$, $\mathrm{p}=0,0012)$. As únicas características que indicaram pequenas inversões não significativas foram atratividade e construtividade.

Tabela 1 - Médias aritméticas das atribuições de características a modelos femininos e masculinos, com e sem cigarro por participantes do sexo masculino e do sexo feminino

\begin{tabular}{llllllllc}
\hline SUJEITO (juiz) & \multicolumn{3}{c}{ MASCULINO } & \multicolumn{3}{c}{ FEMININO } \\
\hline \multicolumn{1}{c}{ Modelo } & \multicolumn{2}{c}{ Mulher } & \multicolumn{2}{c}{ Homem } & \multicolumn{2}{c}{ Mulher } & \multicolumn{2}{c}{ Homem } \\
\hline \multicolumn{1}{c}{ Condição } & com & sem & com & sem & com & sem & com & sem \\
\hline construtividade & 6,32 & 6,28 & 4,64 & 5,88 & $4,28^{*}$ & $7,00^{*}$ & $5,48^{*}$ & $8,16^{*}$ \\
atratividade & 7,64 & 7,60 & 2,40 & 3,60 & 6,56 & 5,72 & 3,48 & 4,16 \\
saúde & $6,08^{*}$ & $8,80^{*}$ & 5,48 & 6,52 & 6,68 & 7,64 & $5,60^{*}$ & $8,32^{*}$ \\
agradabilidade & 6,20 & 7,72 & 3,84 & 5,20 & 5,96 & 5,80 & 5,56 & 7,36 \\
legal & $5,72^{*}$ & $7,88^{*}$ & 4,68 & 5,56 & 5,16 & 5,68 & 5,96 & 7,36 \\
lealdade & $5,12^{*}$ & $7,36^{*}$ & 4,32 & 4,60 & 4,88 & 5,68 & 4,60 & 6,56 \\
pacificidade & 7,36 & 8,92 & 7,12 & 7,96 & 6,56 & 7,88 & 6,44 & 8,60 \\
calma & $5,28^{*}$ & $8,08^{*}$ & 6,92 & 6,92 & $3,68^{*}$ & $6,92^{*}$ & 6,20 & 7,64 \\
otimismo & 5,72 & 6,36 & 6,32 & 7,08 & $7,52^{*}$ & $5,52^{*}$ & 6,48 & 7,84 \\
solidariedade & 6,04 & 6,32 & 5,16 & 6,56 & 5,08 & 5,92 & 6,00 & 7,00 \\
discrição & 6,28 & 7,00 & $3,40^{*}$ & $6,96 *$ & 5,20 & 6,76 & 5,76 & 7,96 \\
confiabilidade & 5,16 & 6,40 & 5,32 & 5,44 & 4,68 & 5,88 & $4,76^{*}$ & $7,60^{*}$ \\
realização & 5,44 & 6,20 & 4,72 & 5,16 & 5,16 & 5,72 & 5,44 & 7,60 \\
humildade & $4,44^{*}$ & $7,60^{*}$ & 4,72 & 5,06 & $3,96^{*}$ & $7,80^{*}$ & 5,72 & 6,04 \\
sabedoria & $5,20^{*}$ & $8,04^{*}$ & 5,04 & 5,96 & $4,80^{*}$ & $5,56^{*}$ & 7,68 & 7,32 \\
\hline
\end{tabular}

Legenda: Prova de Mann-Whitney entre as condições, onde * representa diferença significativa para p < 0,05. Fonte: Dados da pesquisa. 
Aplicando-se a prova de Mann-Whitney às características individualmente, verificamos que as características saúde $(\mathrm{U}=176,0 \mathrm{p}=0,008)$, legal $(\mathrm{U}=$ $192,5 \mathrm{p}=0,0199)$, lealdade $(\mathrm{U}=197,5 \mathrm{p}=0,0257)$, calma $(\mathrm{U}=174,0 \mathrm{p}=0,0072)$, bumildade $(\mathrm{U}=179,0$ $\mathrm{p}=0,0036)$ e sabedoria $(\mathrm{U}=159,0 \mathrm{p}=0,0029)$ apresentaram resultados estatisticamente significativos. As outras características não apresentaram diferença significativa. Isso comprova a nossa hipótese de que os modelos sem cigarro seriam julgados mais positivamente do que os modelos com cigarro.

No julgamento realizado pelos indivíduos do sexo masculino com relação ao modelo masculino percebe-se, a partir das médias, que todas as características $(100 \%)$ foram mais positivas para modelos sem cigarro. O teste estatístico de Wilcoxon, aplicado às características em geral, indicou uma diferença significativa no julgamento $(Z=-2,9505$ $\mathrm{p}=0,0032)$. Tomadas individualmente, verificou-se pelo teste de Mann-Whitney que a única variável que demonstrou diferença significativa foi discrição $(\mathrm{U}=144,5 \mathrm{p}=0,0011)$.

\section{Participantes femininos}

A Tabela 1 permite verificar que a maioria das características $(80 \%)$ atribuídas a modelos femininos apresenta diferença favorável à condição sem cigarro. Analisadas em conjunto, por meio da prova de Wilcoxon, verificou-se uma diferença significativa entre os julgamentos $(Z=-2,3002$ $\mathrm{p}=0,0214)$.

Observando-se os resultados dos julgamentos femininos com relação à mulher, pode-se constatar inversões nos valores de algumas características. No julgamento do modelo feminino para a característica atratividade, o modelo apresentado na condição sem cigarro recebe uma valoração menor do que o com cigarro (não significativa). O mesmo tipo de inversão ocorre para a característica agradabilidade e otimismo, já que nessa última foi possível verificar uma diferença estatisticamente significativa.

Analisadas individualmente, apenas algumas das características evidenciaram diferenças significativas. Tais variáveis foram construtividade $(\mathrm{U}=174,5 \mathrm{p}=0,0074)$, calma $(\mathrm{U}=182,5 \mathrm{p}=$ $0,0117)$, otimismo $(\mathrm{U}=200,5 \mathrm{p}=0,0298)$, bumildade $(\mathrm{U}=153,0 \mathrm{p}=0,0007)$ e sabedoria $(\mathrm{U}=208,5$ $\mathrm{p}=0,0436)$.
No julgamento do modelo masculino, verificou-se que quase todas as variáveis investigadas $(93 \%)$ apresentaram maiores valores para o modelo sem cigarro. A única inversão não significativa ocorreu na característica sabedoria. Quando analisadas em conjunto pela prova de Wilcoxon, verificou-se uma diferença significativa entre a condição com cigarro e sem cigarro $(Z=-3,2942 \mathrm{p}=0,0010)$.

Analisadas individualmente pela prova de Mann-Whitney, verificou-se que apenas as características construtividade $(\mathrm{U}=189,5 \mathrm{p}=0,0170)$, saúde $(\mathrm{U}=193,0 \mathrm{p}=0,0204)$ e confiabilidade $(\mathrm{U}=174,5$ $\mathrm{p}=0,0074)$ apresentaram diferenças estatisticamente significativas, enquanto as outras nada evidenciaram nesse sentido.

\section{Fumantes $\times$ Não fumantes}

Verificou-se, na comparação entre fumantes e não fumantes, que $60 \%$ das características atribuídas aos modelos com cigarro por juízes fumantes apresentam valores ligeiramente maiores. $\mathrm{O}$ teste de Wilcoxon aplicado ao conjunto das características não demonstrou diferença significativa $(Z=-0,3453$ $\mathrm{p}=0,7299)$ no julgamento das características para os mesmos modelos avaliados por sujeitos fumantes e não fumantes.

Analisadas individualmente por meio da prova de Mann-Whitney, nenhuma das características evidenciou diferenças significativas entre os grupos investigados.

\section{Discussão}

Os resultados apresentados demonstram que, de uma forma geral, os adolescentes de ambos os sexos caracterizam os indivíduos fumantes de forma menos favorável.

Tal comprovação evidencia que, do ponto de vista da valorização social, aparentemente, houve um avanço na direção do esclarecimento acerca dos males causados pelo cigarro. Esse processo, ainda que percebido subliminarmente, tende a se materializar em uma diminuição gradual no hábito de fumar entre os adolescentes de onde foi extraída a amostra investigada. Dessa forma, uma análise mais pormenorizada das características se faz necessária. 


\section{Julgamentos masculinos}

A atribuição de características realizada pelos sujeitos masculinos demonstrou um julgamento diferencial entre os modelos apresentados. Enquanto seis características (40\%) evidenciaram diferenças significativas a favor do modelo sem cigarro entre as mulheres, apenas uma $(6,7 \%)$ foi detectada no julgamento dos homens. De uma forma geral, podemos dizer que os sujeitos masculinos são mais rigorosos no julgamento de mulheres do que de homens fumantes.

Algumas das categorias em que as diferenças foram estatisticamente significativas corroboram informações de amplo conhecimento. A característica saúde foi avaliada com maiores valores quando o modelo aparecia sem cigarro. Trata-se do enfoque predominantemente adotado pelas campanhas antitabagistas, evidenciando-se, dessa forma, uma percepção já introjetada nos adolescentes pesquisados. No entanto, cabe lembrar que os jovens nem sempre adotam os comportamentos que sabem ser mais saudáveis (Frankenberger, 2004).

Algumas associações podem ser observadas nos resultados obtidos. É o que se verifica, por exemplo, na questão da saúde e da sabedoria. Com efeito, alguém que atua contra seu próprio organismo não é vista como muito sábia. A bumildade atribuída nesta pesquisa às mulheres fumantes provavelmente se relaciona a uma percepção de menor evidência social.

Uma característica bastante evidente foi a maior calma atribuída à mulher não fumante. Efetivamente, vários estudos realizados demonstraram que as mulheres têm maior dificuldade de abandonar o vício de fumar, provavelmente por desenvolverem uma dependência psicológica maior que os homens, no sentido de diminuírem a ansiedade ao fumar (Fritz, Wider, Hardin \& Horrocks, 2008; Plotnikoff, Berkovitz, Rhodes, Loucaides \& Karunamuni, 2007).

No que diz respeito à atratividade pessoal, podemos afirmar que a mulher não fumante foi considerada mais legal e leal. As características legal e lealdade atingiram maiores escores nos julgamentos masculinos com relação à mulher sem cigarro (Tabela 1) essa percepção, intrinsecamente relacionada à seleção de parceiras, pode refletir preferências quanto a características desejáveis num relacionamento. Dessa forma, as mulheres fumantes parecem ser menos valorizadas na avaliação masculina.
No julgamento masculino de um modelo do mesmo sexo, verificou-se que somente a característica discrição foi estatisticamente significativa a favor do modelo não fumante. Essa informação, aparentemente, demonstra uma percepção social de que o fumante quer chamar a atenção (menos discreto), numa atitude imatura de insegurança. Esta é uma hipótese a ser investigada em maior profundidade.

\section{Julgamentos femininos}

Nos julgamentos realizados por pessoas do sexo feminino, tanto para mulheres como para homens, a característica construtividade apresenta diferença favorável ao modelo sem cigarro. Aparentemente as mulheres são mais exigentes em relação às pessoas, percebendo fumantes como autodestrutivos.

Houve uma inversão significativa nos resultados apresentados para otimismo. Parece que a figura feminina associada ao cigarro apresenta-se, para as mulheres, como mais otimista, provavelmente por negligenciar os riscos, uma vez que o hábito de fumar é sabidamente prejudicial à saúde.

$\mathrm{Na}$ característica calma, percebemos que juízes do sexo feminino apontam modelos sem cigarro como mais calmos, particularmente na mulher. Parece que para os homens o fato de fumar não representa indicativo muito forte de descarga de ansiedade, como parece ocorrer para as mulheres (King, Matacin, Marcus, Bock \& Tripolone, 2000).

Nos julgamentos femininos acerca da mulher, percebemos que, quando apresentada sem cigarro, a modelo era considerada com maior humildade (contrário de arrogante). Esse resultado parece indicar que o hábito de fumar, nas mulheres, é visto como uma forma de não passar despercebida, chamando a atenção para si. Estudos realizados na Austrália demonstraram que mulheres fumantes são mais insatisfeitas com sua própria aparência (King et al., 2000).

Verifica-se que, no julgamento feminino, a mulher apresentada sem cigarro era considerada com mais sabedoria-com efeito, essa característica se relaciona intimamente às informações disponíveis aos estudantes.

\section{Participantes fumantes e náo fumantes}

O fato de não terem sido detectadas diferenças estatísticas entre fumantes e não fumantes acerca 
da atribuição de características a pessoas fumantes (com cigarro) demonstra, pelo menos para a amostra investigada, que o hábito de fumar dos sujeitos não faz com que tais pessoas avaliem fumantes de forma mais favorável, como foi observado por Skalle e Rise (2006).

Esse resultado vai contra nossas expectativas iniciais de que fumantes envolvidos em uma situação de dissonância cognitiva tenderiam a valorizar mais pessoas que compartilham com eles o hábito de fumar, enquanto que não fumantes os veriam de forma menos positiva.

Provavelmente a diferença esperada não ocorreu porque os objetivos da pesquisa não foram esclarecidos para os participantes - portanto, os juízes fumantes não possuíam conhecimento da investigação sobre cigarro. Esse fator pode ter atenuado o conflito cognitivo do sujeito que o levaria à dissonância.

Outra possibilidade explicativa é de que os indivíduos engajados no tabagismo, apesar de incapazes de abandonar o hábito de fumar, percebem-no como nocivo.

\section{Consideraçóes finais}

O desenvolvimento do presente trabalho possibilitou o levantamento de informações importantes sobre os aspectos psicológicos e sociais de adolescentes com relação ao tabagismo. Nosso levantamento bibliográfico demonstrou que tal abordagem é inédita nesse assunto, o que abre inúmeras possibilidades de pesquisa e aplicações práticas no direcionamento de campanhas e materiais didáticos sobre o hábito de fumar voltados ao público adolescente.

Existe um consenso mundial de que a prevenção é sem dúvida o melhor meio de se diminuir as estatísticas de tabagismo, impedindo ou desestimulando os jovens a iniciar o hábito de fumar, uma vez que é muito mais difícil parar o hábito quando este se estabelece, notadamente entre as mulheres (Dedobbeleer et al., 2004; Patel et al., 2004).

No que tange a proibições, verifica-se um esforço reiterado em impedir que os jovens tenham oportunidades de experimentar cigarros por meio de proibições nas escolas (Gilpin et al., 2004) ou que sejam influenciados pelos meios de comunicação com a veiculação de comerciais de apelo infantil (alguns personagens de desenho foram proibidos) ou adolescente (atualmente só podem ser utilizados modelos que aparentam mais de vinte e cinco anos).

A outra vertente relacionada à diminuição do tabagismo entre jovens diz respeito ao desestímulo ao hábito de fumar, realizado por meio das escolas, veiculado nos meios de comunicação na forma de informação científica e, mais recentemente, com algum apelo emocional por meio de fotografias estrategicamente colocadas nos maços de cigarro e, por vezes, em revistas (Machado et al., 2004).

O caminho para as escolas abordarem o assunto nos temas transversais propostos pelos Parâmetros Curriculares Nacionais não pode, de forma alguma, se restringir a informações técnicas e científicas. Embora sejam importantes, elas não atingem os jovens em sua dimensão emocional e de valorização social (Hamilton, Cross, Lower, Resinicov \& Williams, 2003). É necessário que os alunos tenham a oportunidade de manifestar suas opiniões e descobrir o ponto de vista dos outros, que pode não ser favorável ao cigarro. Provavelmente, deve ser muito mais efetiva a informação de que os fumantes são vistos de forma depreciativa, como se evidenciou neste estudo, do que milhares de dados estatísticos sobre doenças causadas pelo fumo.

\section{Apoio financeiro: Bolsa FAPESP}

\section{Referências}

Brasil, F. R., Tavano, L. D., Caramaschi, S., \& Rodrigues, O. M. P. R. (2007). Escolha de parceiros afetivos: Influência das sequelas de fissura labiopalatal. Paidéia, 17(38), 375-387.

Dedobbeleer, N., Beland, F., Contadriopoulos, A. P., \& Adrian, M. (2004). Gender and the social context of smoking behaviour. Social Science \& Medicine, 58(1), 1-12.

Falomir-Pichastor, J. M., Mugny, G., Invernizzi, F., Di-Palma, A., \& Estrada, M. M. (2007). Does the campaign against tobacco use necessarily reduce the attractions of smoking for young people? European Review of Applied Psychology, 57(4), 257-265.

Festinger, L. ([1962] 1975). Teoria da dissonância cognitiva. Rio de Janeiro: Psyche Zahar. 
Forster, J., Chen, V., Blaine, T., Perry, C., \& Toomey, T. (2003). Social exchange of cigarettes by youth. Tobacco Control, 12(2), 148-154.

Fraga, S., Ramos, E., \& Barros, H. (2008). Uso de tabaco por estudantes adolescentes portugueses e fatores associados. Revista de Saúde Pública, 40, 620-626.

Frankenberger, K. D. (2004). Adolescent egocentrism, risk perception, and sensation seeking among smoking and nonsmoking youth. Journal of Adolescent Research, 19(5), 576-590.

Fritz, D.J., Wider,L. C., Hardin, S. B., \& Horrocks, M. (2008). Program strategies for adolescent smoking cessation. The Journal of School Nursing, 24(1), 21-27.

Gibson, B. (1997). Smoker-nonsmoker conflict: Using a social psychological framework to understand a current social controversy. Journal of Social Issues, 53(1), 97-112.

Gilpin, E. A., Lee, L., \& Pierce, J. P. (2004). Does adolescent perception of difficulty in getting cigarettes deter experimentation? Preventive Medicine, 38(4), 485-491.

Gomes, G. R., \& Caramaschi, S. (2007). Valorização de beleza e inteligência por adolescentes de diferentes classes sociais. Psicologia em Estudo, 12(2), 295-303.

Halpern-Felsher, B. L., Biehl, M., Kropp, R., \& Rubinstein, M. L. (2004). Perceived risks and benefits of smoking: Differences among adolescents with different smoking experiences and intentions. Preventive Medicine, 39(3), 559-567.

Hamilton, G., Cross, D., Lower, T., Resinicow K., \& Williams, P. (2003). School policy: What helps to reduce teenage smoking? Nicoyine \& Tobacco Research, 5(1), 507-513.

Hoffman, B. R., Sussman, S., Unger, J. B., \& Valente, T. W. (2006). Peer influences on adolescent cigarette smoking: A theoretical review of the literature. Substance Use and Misuse, 41(1), 103-155.

King, T. K., Matacin, M., Marcus, B. H., Bock, B. C., \& Tripolone, J. (2000). Body image evaluations in women smokers. Addictive Behaviors, 25(4), 613-618.

Machado, S. L., Gomide, P. I. C., Caldeira, R. C. T. (2004). Indústria tabagista e indução ao fumo-os adolescentes percebem a manipulação? In M. Z. S. Brandão \& F. C. S. Conte. (Org.). Sobre o Comportamento e Cognição, 14, 235-241.
Meliska, C. J., \& Gilbert, D. G. (1991). Hormonal and subjective effects of smoking the first five cigarettes of the day: A comparison in male and female. Pharmacology Biochemistry and Behavior, 40, 229-235.

Ogden, J., \& Nicolli, M. (1997). Risk and protective factors: An integration of the epidemiological and psychological approaches to adolescent smoking. Addiction-Research, 5(5), 367-377.

Paek, H. J., \& Gunter, A. C. (2007). How peer proximity moderates indirect media influence on adolescent smoking. Communication Research, 34(4), 407-432.

Patel, J. D., Bach, P. B., \& Kris, M. G. (2004). Lung cancer in US women: A contemporary Epidemic. Journal of the American Medical Association, 291(14), 1763-1768.

Piko, B. F., Bak, J., \& Gibbons, F. X. (2007). Prototype perception and smoking: Are negative or positive social images more important in adolescence? Addictive Behaviors, 32(8), 1728-1732.

Plotnikoff, R. C., Berkovitz, K., Rhodes, R. E., Loucaides, C. A., \& Karunamuni, N. (2007). Testing a conceptual model related to weight perceptions physical activity and smoking in adolescents. Health Education Research, 22(2), 192-202.

Rodriguez, D., \& Audrain-McGovern, J. (2005). Physical activity, global physical self-concept, and adolescent smoking. Annals of Behavioral Medicine, 30(3), 251-259.

Rosendahl, I. K., Galanti, R. M., Gilljam, H., \& Ahlbom, A. (2005). Knowledge about tobacco and subsequent use of cigarettes and smokeless tobacco among Swedish adolescents. Journal of Adolescent Health, 37(3), 224-228.

Simões, M. J. S. (1990). Tabagismo entre estudantes do primeiro e segundo graus em Araraquara, SP. Brasil, 1988. Medicina, 23(4), 223-231.

Silva, M. P., Silva, R. M. V., \& Boelho, C. (2008). Fatores associados à experimentação do cigarro em adolescentes. Jornal Brasileiro de Pneumologia, 34(11), 927-935. 
Skalle, S., \& Rise, J. (2006). The relationship between smoker and nonsmoker prototypes and smoking status among 14-year-old Norvergians. Addictive Behaviors, 31(1), 57-68.

Soldz, S., Huyser, D. J., \& Dorsey, E. (2003). Characteristics of users of cigars, bidis, and kreteks and the relationship to cigarette use. Preventive Medicine, 37(3), 250-258.

Spink, M. J. P. (2009). "Do rato não!”: Materialidades e socialidades na compra de cigarros. Revista Psico, 40(1), 110-120.

Tomar, S. L., \& Hatsukami, D. K. (2007). Perceived risk of harm from cigarettes or smokeless tobacco among U.S High school seniors. Nicotine and Tobacco Research, 9(11), 1191-1196.

White, V., Hill, D., \& Hopper, J. (1996). The outgoing, the rebellious and the anxious: Are adolescent personality dimensions related to the uptake of smoking? Psychology and Health, 12(1), 73-85.

Recebido: 11/02/2010

Received: 02/11/2010

Aprovado: 21/09/2010

Approved: 09/21/2010 